\title{
Suppoting Information for
}

\section{White-Light-Emitting AIE/Eu ${ }^{3+}$-Doped Ion Gel with Multistimuli- responsive Properties}

\author{
Zhehao Tang, Xiaolin Lyu, Longfei Luo, Zhihao Shen,* and Xinghe Fan* \\ E-mail: zshen@pku.edu.cn, fanxh@pku.edu.cn
}

Beijing National Laboratory for Molecular Sciences, Key Laboratory of Polymer Chemistry and Physics of Ministry of Education, College of Chemistry and Molecular Engineering, Peking University, Beijing 100871, P. R. China

Table of Contents

Figure S1. GPC curves of P(TPEE- $b$-EG- $b$-TPEE) and P(TMA-co-MMA) $\quad$ S2

Figure S2. ${ }^{1} \mathrm{H}$ NMR spectrum of P(TPEE- $b$-EG- $b$-TPEE) $\quad$ S2

Figure S3. Luminescent image of the AIE ion gel $\quad$ S2

Figure S4. Excitation spectra of the AIE and $\mathrm{Eu}^{3+}$-doped ion gels $\quad$ S3

Figure S5. Thermogravimetric curves of the AIE and $\mathrm{Eu}^{3+}$-doped ion gels $\quad$ S3

Fiugre S6. ${ }^{1} \mathrm{H}$ NMR spectrum of P(TMA-co-MMA) $\quad$ S3

Figure S7. Luminescent image of the $\mathrm{Eu}^{3+}$-doped ion gel S4

Figure S8. Optical micrographs showing the self-healing process $\quad$ S4

Figure S9. Stability tests of the WLE ion gel S5

Figure S10. $\mathrm{pH}$ responsiveness of the $\mathrm{Eu}^{3+}$-doped ion gel S5

Figure S11. ${ }^{1} \mathrm{H}$ NMR spectra of the protonation of P(TMA-co-MMA) S6

Figure S12. pH responsiveness of the WLE ion gel with DCM S6

Figure S13. SAXS profiles of the AIE ion gel at different temperatures S6

Figure S14. DSC thermogram of P(TPEE- $b$-EG- $b$-TPEE) $\quad$ S7

Figure S15. Cyclic temperature emission spectra of the WLE ion gel S7

Figure S16. Dynamic shear measurements of the hybrid ion gels S8 


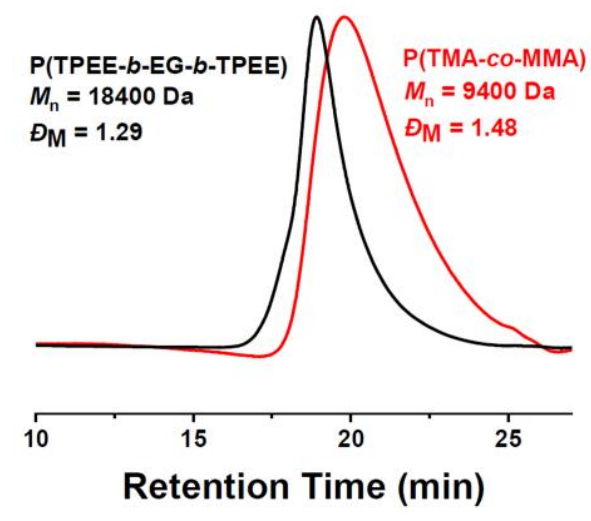

Figure S1. GPC curves of P(TPEE- $b$-EG- $b$-TPEE) and P(TMA-co-MMA).

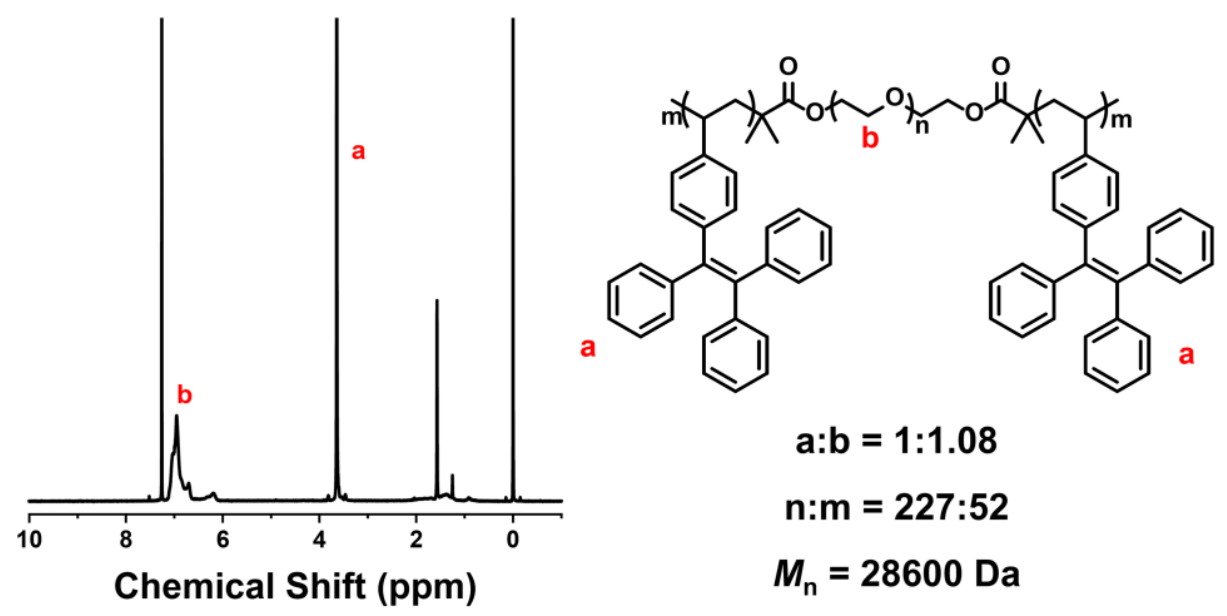

Figure S2. ${ }^{1} \mathrm{H}$ NMR spectrum of $\mathrm{P}\left(\mathrm{TPEE}-b\right.$-EG- $b$-TPEE) with $\mathrm{CDCl}_{3}$ as the solvent.
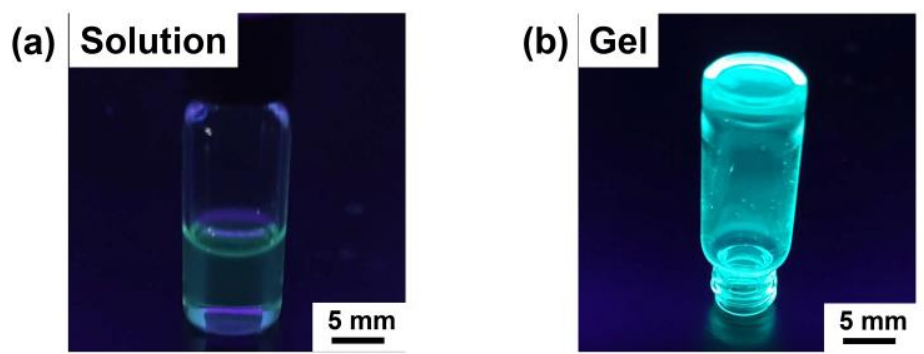

Figure S3. Luminescent images of the P(TPEE- $b$-EG- $b$-TPEE) solution (a) and the AIE ion gel (b). 

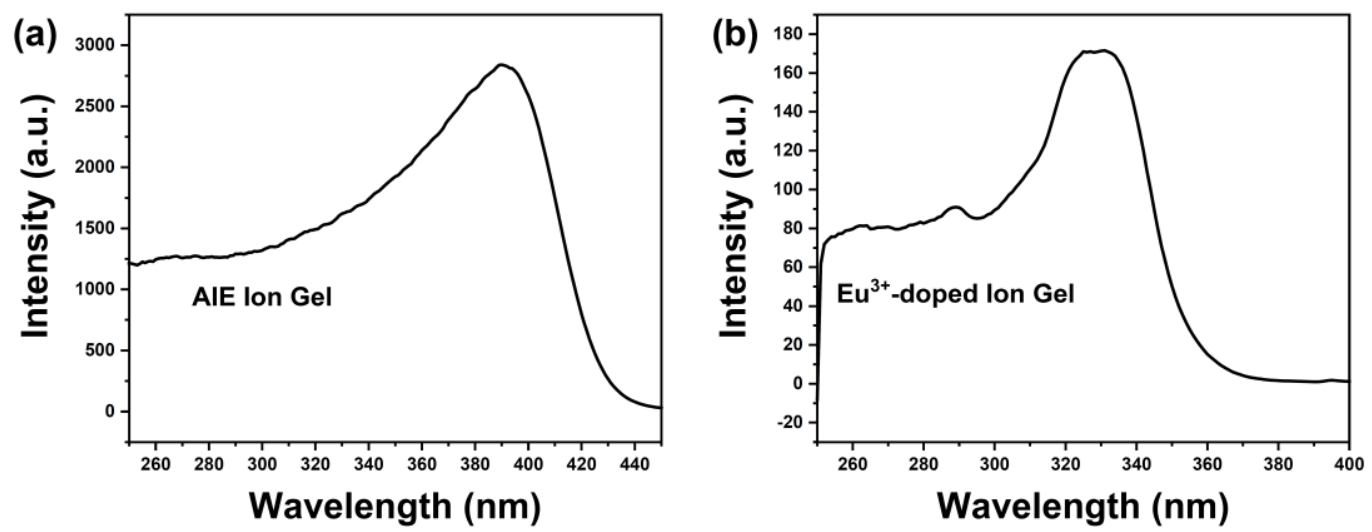

Figure S4. Excitation spectra of the AIE ion gel (a) and the $\mathrm{Eu}^{3+}$-doped ion gel (b).

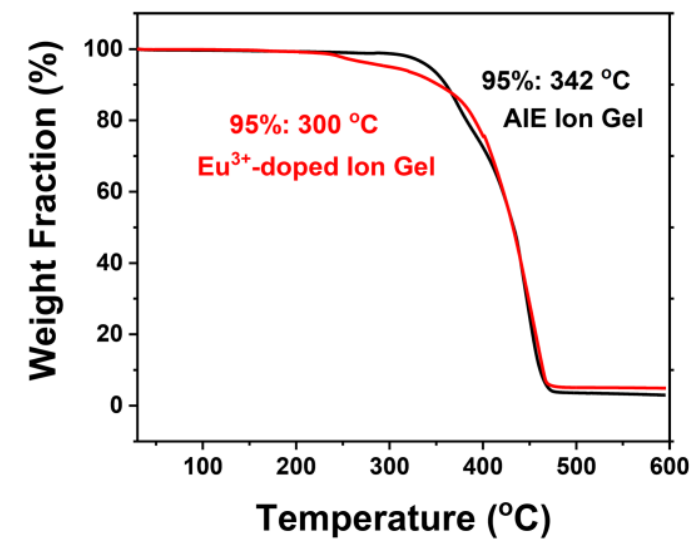

Figure S5. Thermogravimetric curves of the AIE ion gel (black) and the $\mathrm{Eu}^{3+}$-doped ion gel (red) at a heating rate of $10{ }^{\circ} \mathrm{C} / \mathrm{min}$ under nitrogen.

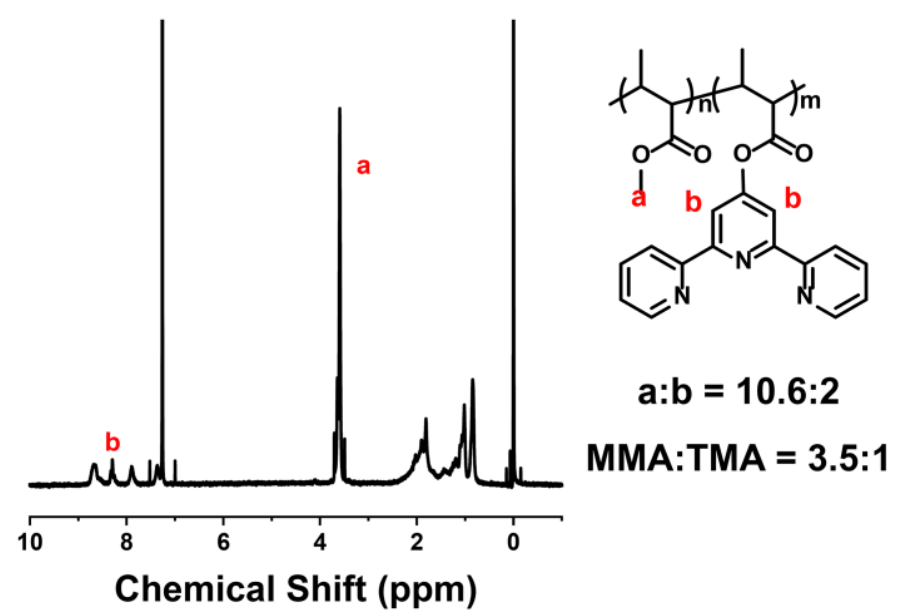

Figure S6. ${ }^{1} \mathrm{H}$ NMR spectrum of $\mathrm{P}(\mathrm{TMA}-c o-\mathrm{MMA})$ with $\mathrm{CDCl}_{3}$ as the solvent. 

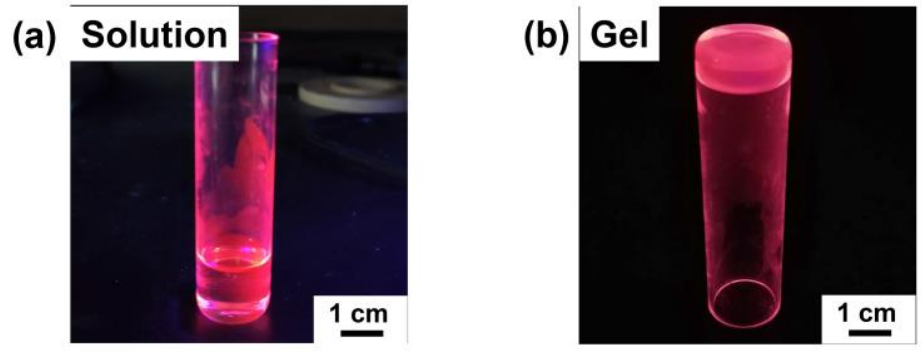

Figure S7. Luminescent images of the $\mathrm{P}(\mathrm{TMA}-\mathrm{co}-\mathrm{MMA}) / \mathrm{Eu}^{3+}$ solution (a) and the $\mathrm{Eu}^{3+}$-doped ion gel (b).

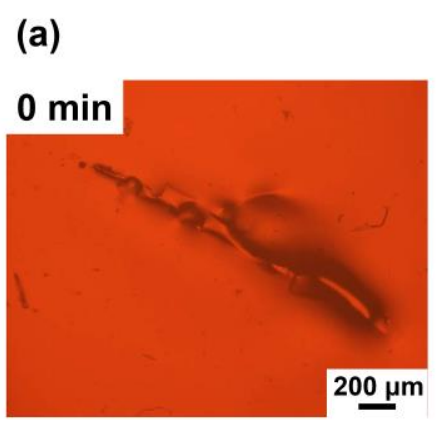

\section{$\mathrm{Eu}^{3+}$-doped Ion Gel}

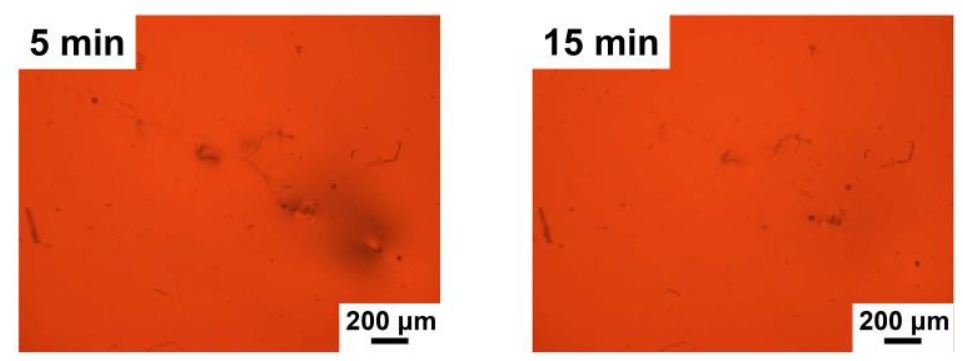

(b)

WLE Ion Gel
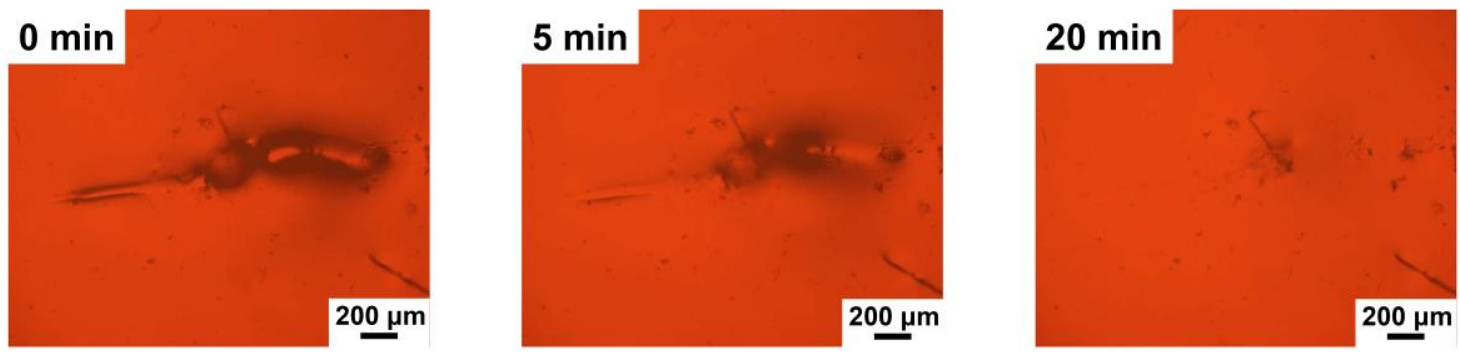

Figure S8. Optical micrographs of the self-healing process of the $\mathrm{Eu}^{3+}$-doped ion gel (a) and the WLE ion gel (b). 


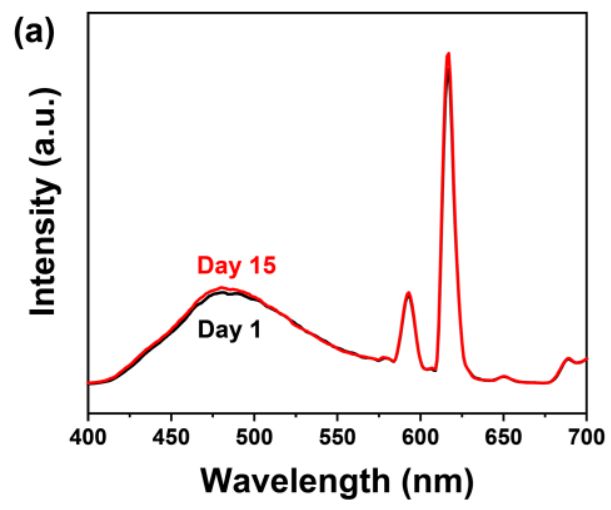

(b)

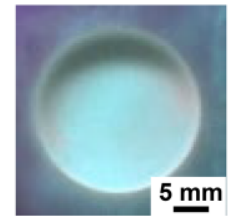

Day 1

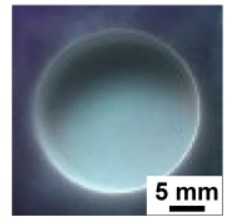

Day 15
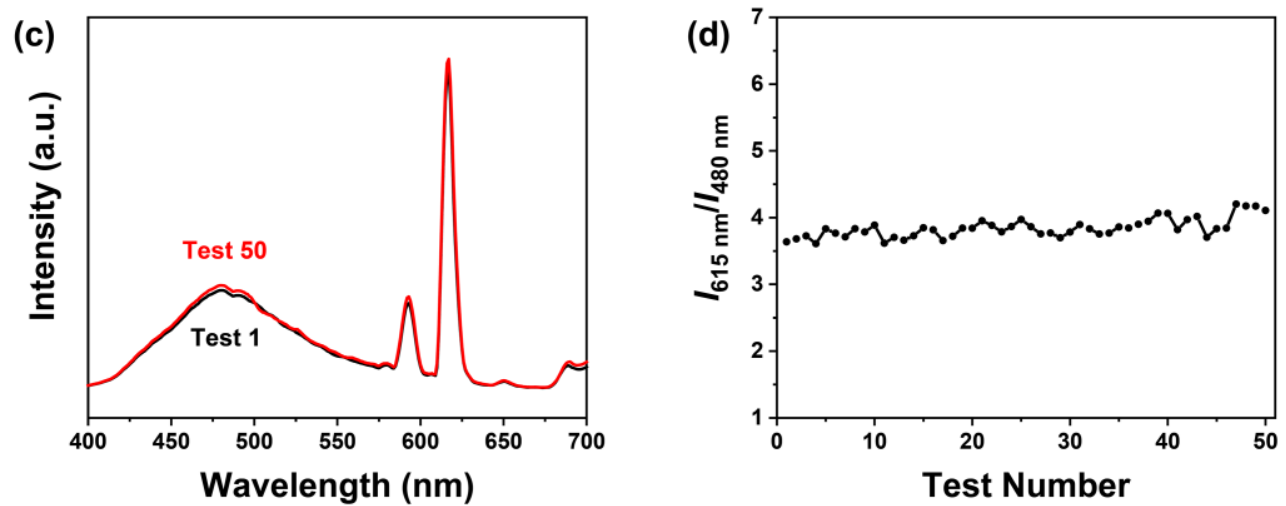

Figure S9. Emission spectra (a) and photographs (b) of the WLE ion gel before and after being placed for 15 days; emission spectra (c) and intensity ratio of the two maximum emission peaks $\left(I_{615 \mathrm{~nm}} / I_{480 \mathrm{~nm}}\right)(\mathrm{d})$ of the WLE ion gel for 50 times of testing.

(a)
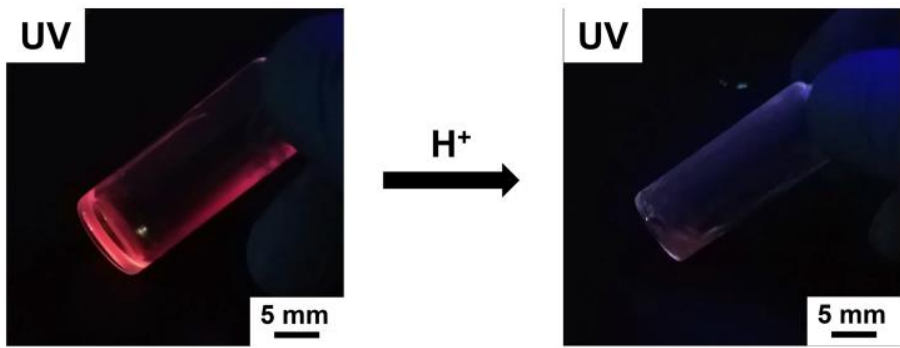

(b)
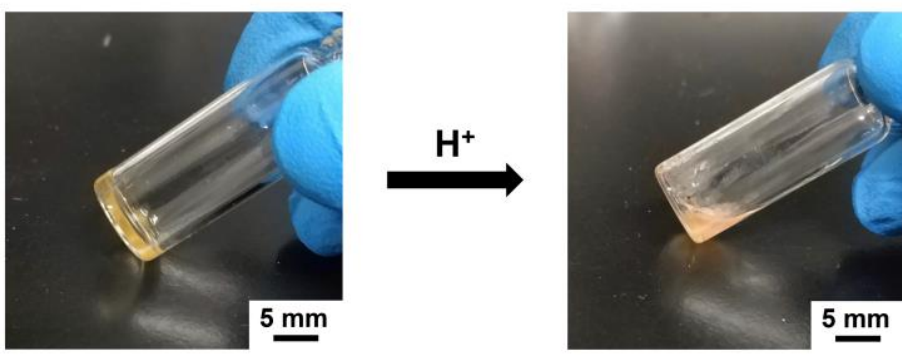

Figure S10. Photographs showing the $\mathrm{pH}$ responsiveness (gel-sol transition and the reductuion of emission intensity) of the $\mathrm{Eu}^{3+}$-doped ion gel (a: under UV irradiation; b: under natrual light). 


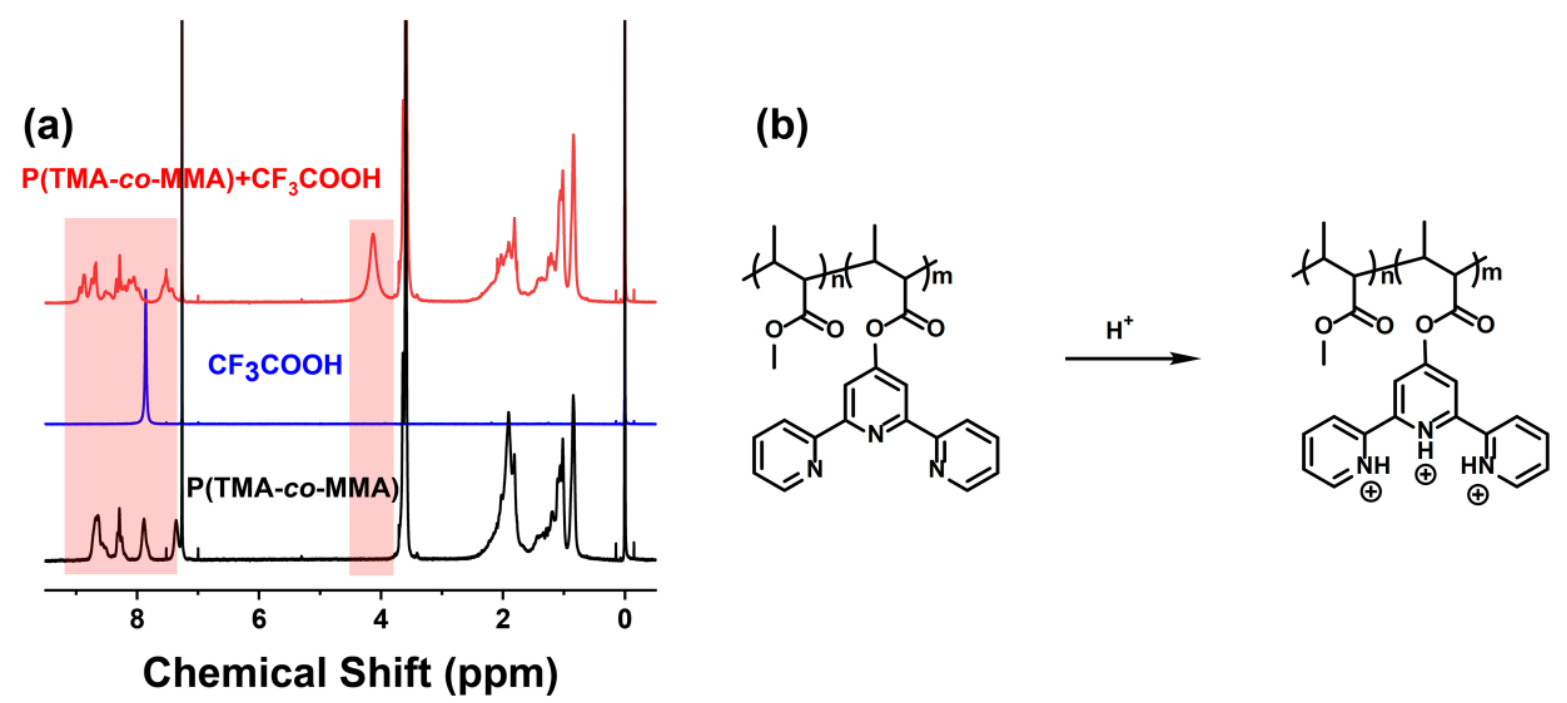

Figure S11. ${ }^{1} \mathrm{H}$ NMR spectra of $\mathrm{P}(\mathrm{TMA}-\mathrm{co}-\mathrm{MMA})$ with and without $\mathrm{CF}_{3} \mathrm{COOH}$ (a) and the scheme of the protonation of P(TMA-co-MMA) (b).
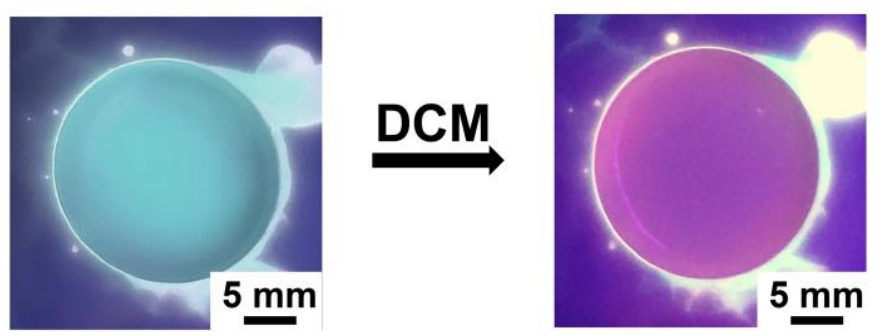

Figure S12. Solvent responsiveness of the WLE ion gel with the addition of DCM.

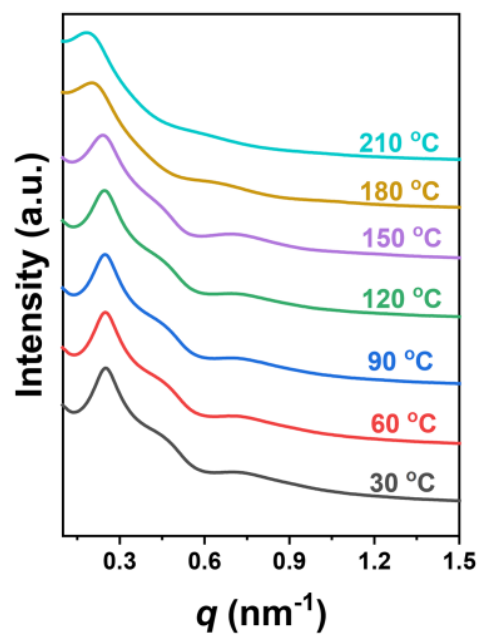

Figure S13. SAXS profiles of the AIE ion gel at different temperatures. 


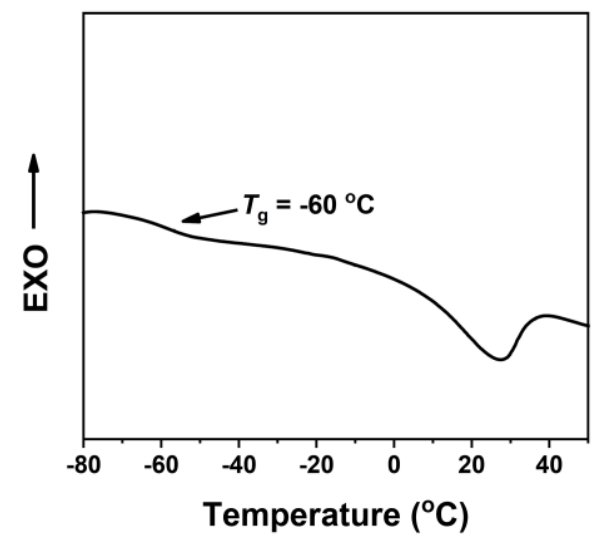

Figure S14. DSC thermogram of P(TPEE- $b$-EG- $b$-TPEE) at a heating rate of $10{ }^{\circ} \mathrm{C} / \mathrm{min}$ under a nitrogen atmosphere.
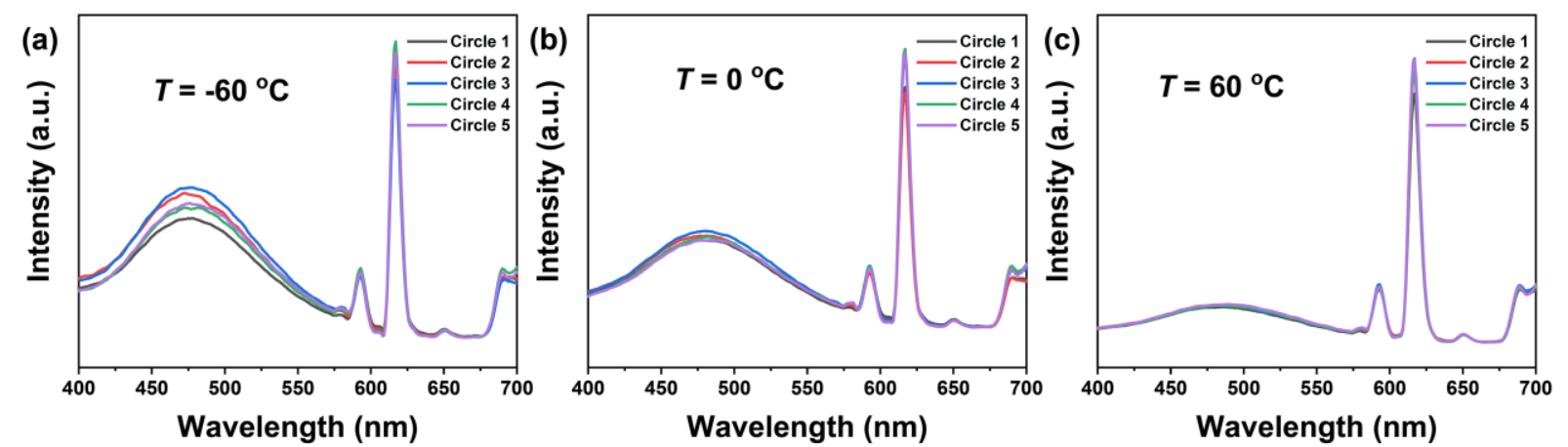

Figure S15. Cyclic temperature responsive emission spectra of the WLE ion gel (a: $-60{ }^{\circ} \mathrm{C}$; b: 0 ${ }^{\circ} \mathrm{C}$; c: $\left.60{ }^{\circ} \mathrm{C}\right)$. 

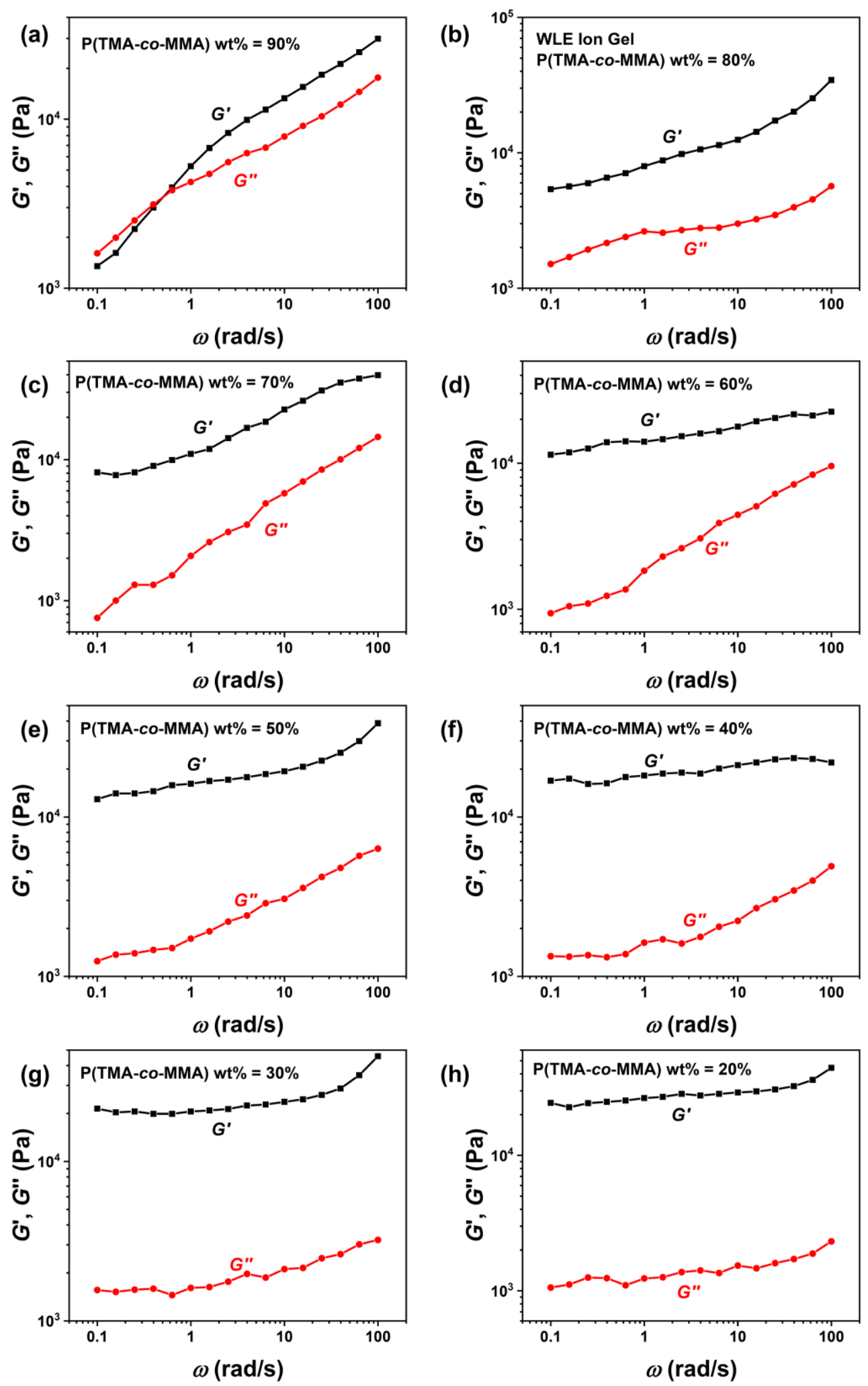

Figure S16. Dynamic shear measurements of the hybrid ion gel with a P(TMA-co-MMA) content of 90 (a), 80 (b), 70 (c), 60 (d), 50 (e), 40 (f), 30 (g), and 20 (h) wt\%. 